\title{
Video as e-learning approach for enhancing laboratory teaching in biochemical engineering- a Malaysia case study
}

\begin{abstract}
In the last decades, evolution of information and communication technologies have been promoting adaptation of new learning and teaching styles. Normal lab execution is conducted using laborious lab manual. Using video visualization as teaching aid together with lab manual in our case is to enhance the teaching and learning of laboratory courses. Furthermore, lack of facilities, staff, resources, fund and time constraint or even scheduling issue may hinder the effectiveness of traditional hands-on experiments by lab manual. This paper describes the design and implementation of two biochemical engineering lab, sterilization intended for students at Bachelor degree. The video is not intended to replace the hands-on experiments using lab manual. Instead its objective is to facilitate further the execution of hands-on experiments by video demonstration. The students were able to watch the hands-on experiments and its procedure prior to the execution of the actual experiments as many times required. The students were exposed to questionnaire at pre-(exposed to lab manual), post- (exposed to lab manual and video), and overall (completed experiments) stage to evaluate cognitive, affective and psychomotor aspects of its implementation. Most of students agreed that the lab video presentation is easier to understand and self-explanatory compared to lab manual alone. This results indicate that video as visualization aid on top of lab manual for experiments execution in biochemical engineering lab is an excellent medium to promote better teaching and learning on student sides. Student becomes more confident, independent, can execute the experiments faster and can relate to the real equipment better. At teachers' or demonstrator side, this video lessened student dependency on them, better impartment and engagement of knowledge with students. On overall, the use of the video promises many positive outcomes particularly to promote TVET based education.
\end{abstract}

Keyword: Video; E-learning; Lab manual; Experiments; Laboratory 